\title{
Reliability Analysis of Molecular Communication Based on Drift Diffusion in Different Topologies
}

\author{
Xinlei Wang ${ }^{*}$, Zhen Jia ${ }^{2}$ \\ ${ }^{1}$ Network Information Center, Xi' an Aeronautical University, Xi'an, China \\ ${ }^{2}$ School of Computer Science, Shaanxi Normal University, Xi'an, China \\ Email: *xleiwang@snnu.edu.cn, jiazhen@snnu.edu.cn
}

How to cite this paper: Wang, X.L. and Jia, Z. (2020) Reliability Analysis of Molecular Communication Based on Drift Diffusion in Different Topologies. Journal of Computer and Communications, 8, 71-89. https://doi.org/10.4236/jcc.2020.81005

Received: December 13, 2019

Accepted: January 13, 2020

Published: January 16, 2020

Copyright $\odot 2020$ by author(s) and Scientific Research Publishing Inc. This work is licensed under the Creative Commons Attribution International License (CC BY 4.0).

http://creativecommons.org/licenses/by/4.0/

\section{(c) (i) Open Access}

\begin{abstract}
Molecular communication is a novel nanoscale communication method. It can use ions, biochemical molecules or other information carriers to transmit information. However, due to molecules are easily accumulated in the channel to produce strong internal symbol interference, the information transmission in the channel is vulnerable to a low reliability. Therefore, reliability has become an important research issue in the field of molecular communication. At present, the existing reliability model of molecular communication does not take into account the drift velocity of the medium. Nevertheless, in some scenarios, it is often necessary to consider the effect of the drift velocity of the medium on the reliability of molecular communication. In this paper, we introduce the drift velocity of the medium and propose a reliability model of molecular communication based on drift diffusion (MCD2) in different topologies. Furthermore, in the case of transmission failure, a retransmission mechanism is used to ensure reliable transmission of information. Finally, we also compare the reliability performance of molecular communication between reliability model of MCD2 and reliability model of molecular communication based on free diffusion. The simulation results indicate that the proposed reliability model is superior to the existing reliability model of molecular communication based on free diffusion in analyzing the reliability of MCD2.
\end{abstract}

\section{Keywords}

Nanonetwork, MCD2, Drift Diffusion, Reliability

\section{Introduction}

In recent years, with the rapid development of the Internet and 5G communica- 
tion technologies, people increasingly depend on network communication technologies. At the level of micro-network communication outside the traditional communication network, nanocommunication technologies with different mechanisms have received extensive attention from researchers.

There are two main methods for implementing nanonetworks, namely bio-inspired molecular communication and electromagnetic nanocommunication. Bioinspired molecular communication generally uses chemical signals, cells, organelles or DNA as carriers. As for electromagnetic nanocommunication generally uses carbon nanotubes as a carrier. With the development of nanonetwork research, multiple nano-sensors can be deployed in the human body to monitor the substances in the human body, such as glucose, sodium and cholesterol. Then these sensors can transmit detection information to mobile phones or computers to dynamically display the health of the human body. Nano-sensors can also detect the specific parts of human infections to prevent human diseases. To achieve the above functions, it must rely on tiny devices with bionic functions, such as nanomachine. Nanomachine can be made up of nanoscale components that perform tasks such as calculation, storage, sensing, and driving. At present, there are three different ways to make nanomachine [1], such as top-down method, a bottom-up method, and a biological hybrid method.

However, due to the limited task types and space scope of a single nanomachine, in order to complete more complex tasks in a larger scope, it is necessary to connect multiple nanomachines to form a nanonetwork for task collaboration and information sharing. To the best of our knowledge, there are four ways to achieve communication between nanomachines, nanomechanical communication, acoustic communication, electromagnetic communication, and molecular communication. Among the above four communication methods, researchers have found that molecular communication is the most likely way to achieve communication between nanomachines. This mainly depends on bio-inspired molecular communication, which generally uses chemical signals, cells or organelles, and DNA as vectors to transmit information to adapt to functional characteristics of nanomachine.

At present, the main goal in the field of molecular communication is to enable nanomachine can communicate with each other in a biological environment. In this new mode of communication, information can be encoded as a carrier. such as a molecule or ion for propagation. Examples of using molecular communication to solve problems including calcium ion signals [2], molecular diffusion [3], bacterial communication [4]. Therefore, it can be applied to a wide variety of fields. For example, the military field, the industrial field, the environmental field [5], and the biomedical field [6].

Molecular communication is a new communication technology. It can use bioinformatics molecules or ions to encode different information for transmission. However, the controllability and reliability of molecular communication are low due to the interference between various symbolic molecules and the 
random walk of molecules. Therefore, the reliability of molecular communication has become an important research direction in the field of molecular communication. Nevertheless, the reliability research of existing molecular communication has mainly involved the reliability analysis of molecular communication in free diffusion channels, without considering the influence of the drift velocity of the medium on the reliability of molecular communication. In this paper, a reliability model of molecular communication based on drift diffusion is proposed on the premise of drift velocity.

In the reliability research of molecular communication, Frank et al. [7] used virus particle as information carrier to study the reliability and delay of multi-hop molecular communication. Balasubramaniam et al. [4] analyzed the characteristics of multi-hop nanonetworks, such as the reliability of information transmission under different topologies. Cheng et al. [8] have studied the reliability and delay of multicast topology under binary communication. Einolghozati et al. [9] have studied the reliability problem between two nodes in a bacterial colony molecular communication network. Tepekule et al. [10] have proposed two different types messenger molecules to reduce the impact of ISI to improve the reliability of molecular communication. Shih et al. [11] used new channel code techniques to improve the reliability of molecular communication. Leeson et al. have used error correction code to reduce the decode error rate and improve the reliability of the communication link [12]. Based on the above reliability analysis of molecular communication, these researches have not mentioned the influence of the drift velocity on the reliability of molecular communication. Nevertheless, in our previous laboratory, Lu et al. [13] [14] considered the drift velocity of medium, they proposed the molecular communication model in the vertical direction and its test bed, but no further involves the reliability of molecular communication.

The motivation of this paper is to analyze the reliability of molecular communication on the premise of drift velocity. Based on this goal, we propose a reliability model of molecular communication based on drift diffusion under different topologies. In the case of a transmission failure, a retransmission mechanism is used to ensure reliable transmission of information.

\section{1) Our Contribution}

- According to the existing research of molecular communication reliability, we introduced the drift velocity of the medium to propose a reliability model of molecular communication based on drift diffusion under different topologies.

- In the case of transmission failure, a retransmission mechanism is used to ensure reliable transmission of information.

- In addition, we also analyze the influence of different model parameters and different topologies on the reliability of MCD2, which can guide us to select optimal parameter and topology to improve the reliability of molecular communication. 


\section{2) Paper Outline}

The rest of this paper is organized as follows. Section 2 a system model can be described. In Section 3, we proposed the reliability model of MCD2 in the single link, single path and multipath. Simulation results and analysis are presented in Section 4. Finally, we summarize this paper in Section 5.

\section{System Model}

In this section, as shown in Figure 1, a system of molecular communication based on drift diffusion is described as consisting of the following subsystems.

\section{- Transmitter nanomachine (TN)}

The transmitter nanomachine can continuously generate the same molecules to transmit information. Assuming that the TN can precisely control the release time of the molecule, and once these molecules are released into the channel by the TN, they will no longer be affected by the TN.

\section{- Transmission medium}

In a fluid medium, information molecules propagate information between a $\mathrm{TN}$ and a receiver nanomachine.

\section{- Receiver nanomachine (RN)}

When these molecules arrive at the $\mathrm{RN}$, the $\mathrm{RN}$ can decode the information from the TN. Then, these molecules can be completely removed from the current channel by the RN.

Here, it is supposed that the TN and the RN are highly synchronized at all times and that the information molecules only fully elastically collide with the $\mathrm{RN}$ surface in the drift diffused channel. At the same time, the diffusion motion of the information molecules released by the TN, which can be attributed to the one-dimensional Brownian motion in the forward direction of the drift velocity to simplify the analysis process.

MCD2 has great application prospects in the field of biomedicine. For example, in the human body, cell to cell communication can through the diffusion of some information molecules or ions to transmit information. This process can be abstracted as a communication process between two nanomachines. The communication system model can be described as Figure 1 .

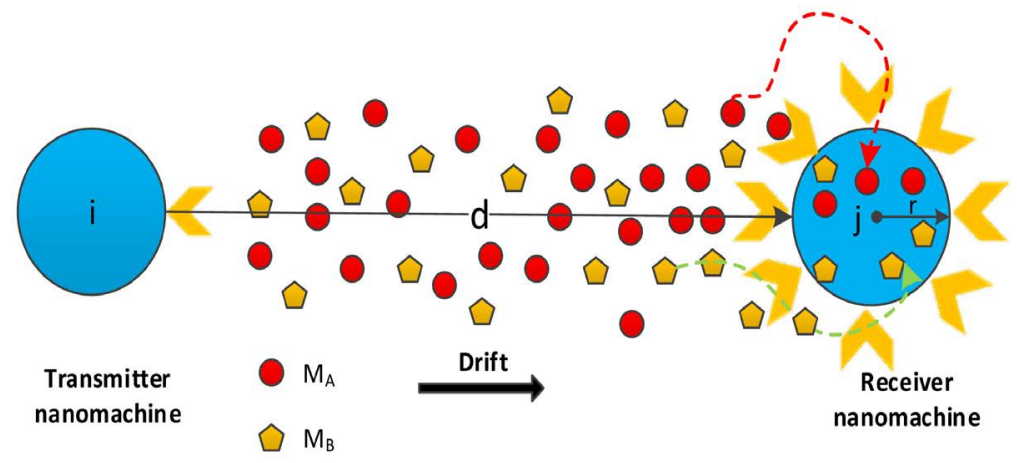

Figure 1. The system of molecular communication based on drift diffusion. 
As shown in Figure 1, when the TN releases the information molecules into the channel, after a period of time, the information molecules reach the RN by the drift diffusion, and the $\mathrm{RN}$ will decode the received information molecules to obtain the original information.

It can be seen from Figure 2, under the channel of MCD2, the communication process mainly includes the five processes, which are modulation, send, transmission, receive and demodulation, respectively.

It is assumed that the transmission of information between the TN and the $\mathrm{RN}$ is mainly based on the binary sequence. In order to avoid mutual interference of the same type of molecules, we use two different types of molecules $\mathrm{A}$ and $B$ to represent " 1 " and " 0 ", respectively. Then, we use the array $a[i]$ to represent the information transmitted each time. And the encode of the information by the TN can be formally defined as follows:

$$
a[i]=\left\{\begin{array}{l}
1, \mathrm{TN} \rightarrow M_{A} \\
0, \mathrm{TN} \rightarrow M_{B}
\end{array}\right.
$$

Since the motion of the information molecules in the channel is affected by the Brownian motion, the diffusion process is random, and the transmission time of these molecules to the RN is also random. Without considering the drift velocity, the information molecules propagate in the form of Brownian motion in the channel. According to Fick's second law, the partial derivative of the concentration of information molecules with respect to time is expressed as follows [15]:

$$
\frac{\partial c(t)}{\partial t}=D \times \frac{\partial^{2} c(t)}{\partial x^{2}}
$$

Therefore, under the molecular communication channel based on free diffusion, for any information molecule released by the TN at time $t=0$, the probability of the molecule at different position $x$ can be used $P(x, t)$ to calculate, which its expression is as follows:

$$
P(x, t)=\frac{1}{\sqrt{4 \pi D t}} \exp \left(-\frac{x^{2}}{4 D t}\right)
$$

In the molecular communication channel based on drift diffusion, it is

Transmitter nanomachine

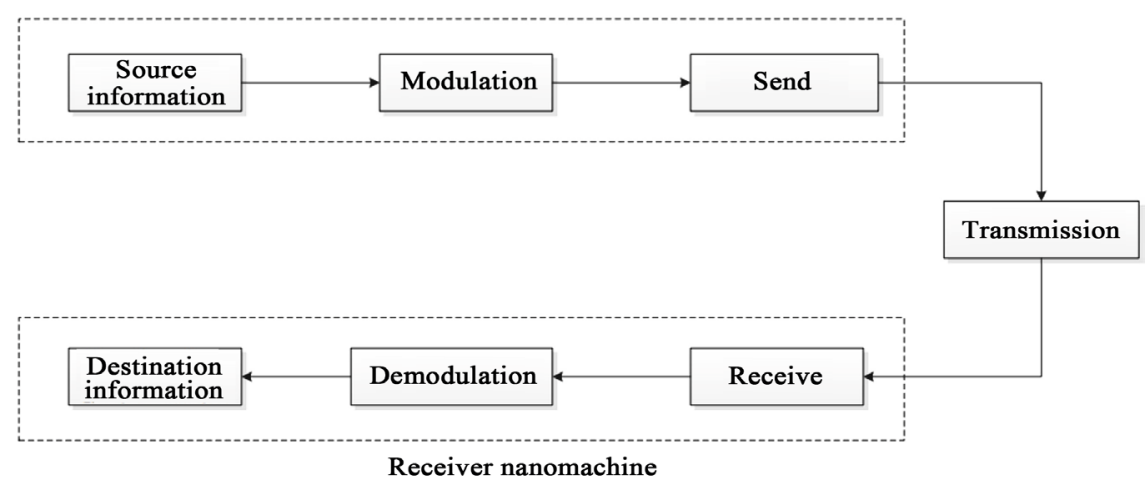

Receiver nanomachine

Figure 2. Basic process of molecular communication based on drift diffusion. 
necessary to consider the influence of the drift velocity of the medium on the impulse response of the system. We suppose that the drift velocity only consists of the positive drift velocity in the horizontal direction. In this paper, we only consider the propagation of information molecules in the drift diffusion channel in the one-dimensional case. Therefore, according to Fick's second law, under the drift diffusion channel, the partial derivative relationship of the concentration $c(t)$ of the information molecule with respect to time $t$ is as follows:

$$
\frac{\partial c(t)}{\partial t}=D \times \frac{\partial^{2} c(t)}{\partial x^{2}}-v_{x} \frac{\partial c(t)}{\partial x}
$$

In this paper, we suppose that the $\mathrm{RN}$ does not have an absorption boundary under the flow medium, ranging from negative infinity to positive infinity. For any information molecule released by the TN at time $t=0$, the probability of the molecule at different position $x$ can be calculated by using the position distribution function $g(x, t)$ [16], which is expressed as follows:

$$
g(x, t)=\frac{1}{\sqrt{4 \pi D t}} \exp \left(-\frac{\left(x-v_{x} t\right)^{2}}{4 D t}\right)
$$

In one dimensional environment, the position of any information molecule changes with time, which obeys the position probability density function $g(x, t)$. Using this distribution, we can get that the time of any information molecule released by the TN reaches the $\mathrm{RN}$ after the moving $d$ distance obeys the probability density function $f(t)$. Here we make the horizontal drift velocity $\left(v_{x}=v\right)$, and its function expression $f(t)$ is as follows:

$$
f(t)=\frac{d}{\sqrt{4 \pi D t^{3}}} \exp \left(-\frac{(d-v t)^{2}}{4 D t}\right), t>0
$$

In the above Equation (5), $D$ represents the diffusion coefficient of medium and $v$ represents the drift velocity of the medium. According to the function expression of $f(t)$, the cumulative distribution function $F(t)$ can be obtained, which represents the probability any information molecules generated by the TN reaches the $\mathrm{RN}$ before time $t$. Then, the calculation expression of $F(t)$ is as follows:

$$
F(t)=\frac{1}{2}\left(1+\operatorname{erf}\left(\sqrt{\frac{d}{4 D t}}\left(\frac{v t}{d}-1\right)\right)\right)+\frac{1}{2} \exp \left(\frac{v d}{D}\right)\left(1+\operatorname{erf}\left(-\sqrt{\frac{d}{4 D t}}\left(\frac{v t}{d}+1\right)\right)\right) .
$$

\section{Reliability Analysis of MCD2}

In this section, we will give a mathematical model of reliability of MCD2 in single link, and then we also extend this reliability model into single path and multipath.

\subsection{The Analysis of Reliability in Single Link}

If there is no other relay node between the $\mathrm{TN}$ and the $\mathrm{RN}$, this transmission path is defined as a single link. As shown in Figure 1, we present a system model 
of MCD2 under a single link. At the same time, we assume that the time required for the TN to transmit one bit of information is $T$, which transmission time $T$ can be divide into $m$ equal time slots $\tau$, that is $T=m \tau$. When the TN transmits bit information " 1 " in a time slot, the probability of the event is recorded as $P\left(s_{A}=1\right)$. Similarly, when the TN transmits the bit information " 0 " in a time slot, the probability of the event is recorded as $P\left(s_{A}=0\right)$. Then the following Equation can be established:

$$
P\left(s_{A}=1\right)+P\left(s_{A}=0\right)=1
$$

We suppose that TN transmits bit information "1" or " 0 " with the same channel transmission probability of $\lambda$, the above Formula (7) can be reduced to $2 \lambda=1$. Namely, $\lambda=0.5$.

When time slot $k \in\{1,2,3, \cdots, n-1\}$, TN transmits the information molecules with the channel transmission probability of $\lambda$ without being received by $\mathrm{RN}$, the probability that this molecule reaches the $\mathrm{RN}$ in the $n^{\text {th }}$ time slot is recorded as $\gamma_{i j}(k, n)$, and its calculation expression is as follows:

$$
\begin{aligned}
\gamma_{i j}(k, n) & =\lambda \int_{(n-k) \tau}^{(n-k+1) \tau} f(t) \mathrm{d} t \\
& =\lambda[F((n-k+1) \tau)-F((n-k) \tau)] \\
& =\frac{1}{2}[F((n-k+1) \tau)-F((n-k) \tau)], n>k
\end{aligned}
$$

where $F(\tau)$ is given in Equation (6). And when $n=k, \gamma_{i j}(n, n)$ can be calculated by $\gamma_{i j}(n, n)=0.5 F(\tau)$.

According to the above analysis, the probability that $\mathrm{M}$ molecules of type $\mathrm{A}$ or $\mathrm{B}$ released by the TN are not received by the $\mathrm{RN}$ in the $n^{\text {th }}$ time slot is represented by $\beta_{i j}$, and the calculation expression is as follows:

$$
\beta_{i j}=\prod_{k=1}^{n}\left(1-\frac{1}{2}[F((n-k+1) \tau)-F((n-k) \tau)]\right)^{M}
$$

The reliability of MCD2 under a single link is defined as the probability that at least one information molecule released by the $\mathrm{TN}$ is successfully received by the RN before time $\mathrm{T}$, which is recorded as $P_{i j}$. In summary, the calculation expression of the reliability of MCD2 in a single link is as follows:

$$
P_{i j}=1-\prod_{n=1}^{m} \prod_{k=1}^{n}\left(1-\frac{1}{2}(F((n-k+1) \tau)-F((n-k) \tau))\right)^{M}
$$

where $M$ represents the total number of A type or B type information molecules released by the TN in each time slot, and $\mathrm{m}$ represents the number of time slots.

In the case of transmission failure, we use the automatic repeat request mechanism (ARQ) to ensure the reliable transmission of information. The working mechanism is as shown in Figure 3, that is, when the $\mathrm{M}$ information molecules of type A or B released by TN are not successfully received by the RN within a specific time slot. Within the time set by both sides of communication, the TN will continue to regenerate $M$ information molecules releasing in the channel until the $\mathrm{RN}$ receives the information molecules released from the $\mathrm{TN}$, 


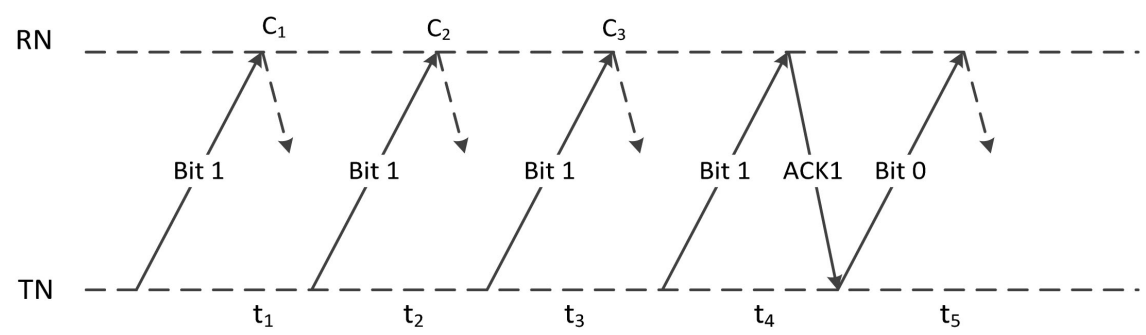

Figure 3. Schematic diagram of automatic retransmission request mechanism for MCD2.

and RN will generate $M$ acknowledgment information molecules for transmission to the TN. Therefore, when the TN receives the confirmation molecule transmitted from the RN, the transmission of one bit information is successfully completed. Here, it is assumed that information molecules and confirmation information molecules are different, but they have the characteristics of common diffusion.

As shown in Figure 3, it is assumed that the maximum number of retransmissions of single link is $C_{i j}$ during the retransmission process. Therefore, after using the automatic retransmission request mechanism, the probability that the $\mathrm{RN}$ receives at least one molecule is denoted as $P_{i j}^{\prime}$, and its calculation expression is as follows:

$$
\begin{aligned}
P_{i j}^{\prime} & =P_{i j}+\left(1-P_{i j}\right) P_{i j}+\left(1-P_{i j}\right)^{2} P_{i j}+\left(1-P_{i j}\right)^{3} P_{i j}+\cdots+\left(1-P_{i j}\right)^{C_{i j}} P_{i j} \\
& =\sum_{n=0}^{C_{i j}}\left(1-P_{i j}\right)^{n} P_{i j}
\end{aligned}
$$

\subsection{The Analysis of Reliability in Single Path}

A single path with two links is described in Figure 4. There is only one relay nanomachine between the TN and the RN.

As can be seen from Figure 4, the communication system consists of TN, relay nanomachine and $\mathrm{RN}$. At the same time, $\mathrm{TN}$, relay nanomachine and $\mathrm{RN}$ are placed in a row. Then in single path communication, we use the same type of molecule to transfer information from TN to RN. In this process, we believe that TN can release $M$ molecules in the channel to transmit a bit information to the relay nanomachine. Then, these information molecules arrive at relay nanomachine which can continue to replicate $M$ molecules by forwarding these information molecules to RN using the same type of molecule. Then, these information molecules can bind to the corresponding receptors from $\mathrm{RN}$, and the information molecules are eventually decoded by RN, while the decoded molecules are removed from the current environment. This means that the communication is successfully completed in the single path.

This communication process can be thought of as two processes. In the first stage, TN releases these information molecules to the relay nanomachine, and from TN to relay nanomachine any molecule experienced by the time $t$ obey $f_{1}(t)$, it represents the probability density function (PDF), it can be with the Equation (5), then $f_{l}(t)$ can be described as the following Equation (12): 


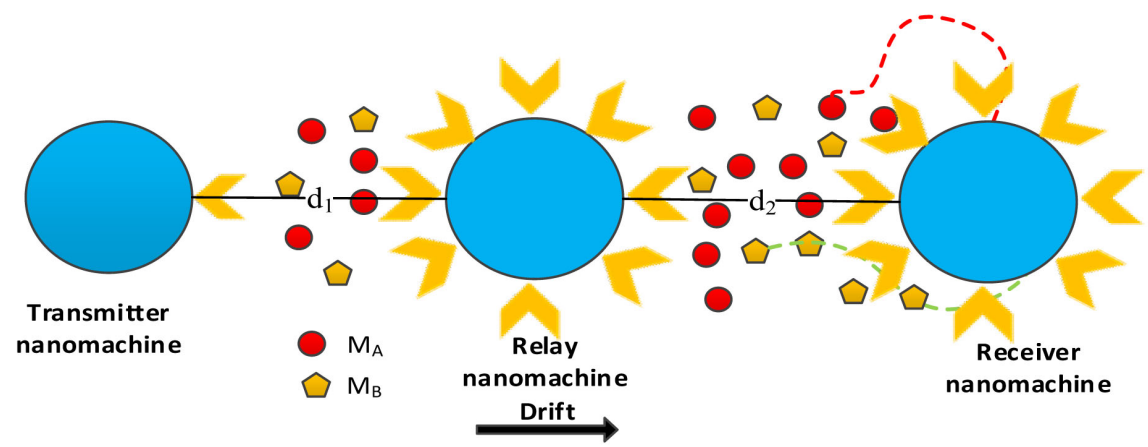

Figure 4. The system of MCD2 in the single path.

$$
f_{1}(t)=\frac{d_{1}}{\sqrt{4 \pi D t^{3}}} \exp \left(-\frac{\left(d_{1}-v t\right)^{2}}{4 D t}\right), t>0
$$

The CDF is denoted by $F_{1}(t)$ which could be connected with the probability density function $f_{1}(t)$ in Equation (12) as follows Equation (13):

$$
F_{1}(t)=\frac{1}{2}\left(1+\operatorname{erf}\left(\sqrt{\frac{d_{1}}{4 D t}}\left(\frac{v t}{d_{1}}-1\right)\right)\right)+\frac{1}{2} \exp \left(\frac{v d_{1}}{D}\right)\left(1+\operatorname{erf}\left(-\sqrt{\frac{d_{1}}{4 D t}}\left(\frac{v t}{d_{1}}+1\right)\right)\right)
$$

According to Equation (12), (13), then we can use $P_{1}$ to represent the reliability of communication between the TN and the relay nanomachine. Then $P_{1}$ can be calculated as follows Equation (14):

$$
P_{1}=1-\prod_{n=1}^{m} \prod_{k=1}^{n}\left(1-\frac{1}{2}\left(F_{1}((n-k+1) \tau)-F_{1}((n-k) \tau)\right)\right)^{M}
$$

Similarly, when these information molecules are released by the relay nanomachine first hit the RN. Then we could consider that the time $t$ experienced by any molecule from relay nanomachine to $\mathrm{RN}$ obeys the $f_{2}(t)$, which could be associated with Equation (5), then $f_{2}(t)$ is described as follows Equation (15):

$$
f_{2}(t)=\frac{d_{2}}{\sqrt{4 \pi D t^{3}}} \exp \left(-\frac{\left(d_{2}-v t\right)^{2}}{4 D t}\right), t>0
$$

At the same time, the CDF is denoted by $F_{2}(t)$ which could be connected with the probability density function $f_{2}(t)$ in (15) as follows Equation (16):

$$
F_{2}(t)=\frac{1}{2}\left(1+\operatorname{erf}\left(\sqrt{\frac{d_{2}}{4 D t}}\left(\frac{v t}{d_{2}}-1\right)\right)\right)+\frac{1}{2} \exp \left(\frac{v d_{2}}{D}\right)\left(1+\operatorname{erf}\left(-\sqrt{\frac{d_{2}}{4 D t}}\left(\frac{v t}{d_{2}}+1\right)\right)\right)
$$

According to Equations (15), (16), then we can use $P_{2}$ to represent the reliability of communication between relay nanomachine and the RN. Then $P_{2}$ can be computed as follows Equation (17):

$$
P_{2}=1-\prod_{n=1}^{m} \prod_{k=1}^{n}\left(1-\frac{1}{2}\left(F_{2}((n-k+1) \tau)-F_{2}((n-k) \tau)\right)\right)^{M}
$$

As for the process of the TN releases information molecules to relay nanomachine and then relay nanomachine simultaneously forward these information molecules to $\mathrm{RN}$. We consider that no link failure from $\mathrm{TN}$ to $\mathrm{RN}$ in the single 
path. Then we get the reliability of the single path $P_{3}$ can be denoted as follows Equation (18):

$$
P_{3}=P_{1} P_{2} \text {. }
$$

\subsection{The Analysis of Reliability in Multipath}

In this section, we will investigate the reliability of MCD2 in the multipath. As you can see from Figure 5, the multipath consists of one TN, 4n relay nanomachines and n Receiver nanomachines. As shown from Figure 5, when TN wants to transmit a single bit information by releasing $M$ molecules in each time slot. As for each hop, a released molecule can only attach to the next hop node for forward transmission. The aim is to ensure that at least one information molecule can be successfully received by $\mathrm{RN}_{1}, \mathrm{RN}_{2}$ or $\mathrm{RN}_{\mathrm{n}}$ before time $T$. There are $2 \mathrm{n}$ paths in the $S$ which include TN-M $-\mathrm{M}_{1}-\mathrm{RN}_{1}, \mathrm{TN}-\mathrm{M}_{2}-\mathrm{N}_{2}-\mathrm{RN}_{1}, \mathrm{TN}-\mathrm{M}_{3}-\mathrm{N}_{3}-\mathrm{RN}_{2}$, TN-M $-\mathrm{M}_{4}-\mathrm{N}_{4}-\mathrm{RN}_{2}, T N-\mathrm{M}_{\mathrm{i}}-\mathrm{N}_{\mathrm{i}}-\mathrm{RN}_{\mathrm{n}}$ and TN-M $\mathrm{M}_{\mathrm{n}}-\mathrm{N}_{\mathrm{n}}-\mathrm{RN}_{\mathrm{n}}$. These paths are labeled as $s_{1}, s_{2}, s_{3}, s_{4}, s_{i}, \cdots, s_{2 n}$, which are all disjoint and independent. On the one hand, we define $S$ as the set of the multipath and each path $s_{k}$ has 3 hops. Then set $S$ can be assigned as $S=\left\{s_{1}, s_{2}, s_{3}, s_{4}, s_{i}, \cdots, s_{2 n}\right\}$. On the other hand, we also define a set of corresponding link reliabilities $P_{s_{k}}=\left[P_{i j}, P_{j l}, P_{l m}, \cdots, P_{m 2 n}\right]$, where $P_{i j}$ is the reliability between node $i$ and $j$.

From Figure 5, we can see that the information molecules originate from TN can pass along the paths $s_{1}$ or $s_{2}$ arriving at the destination nanomachine

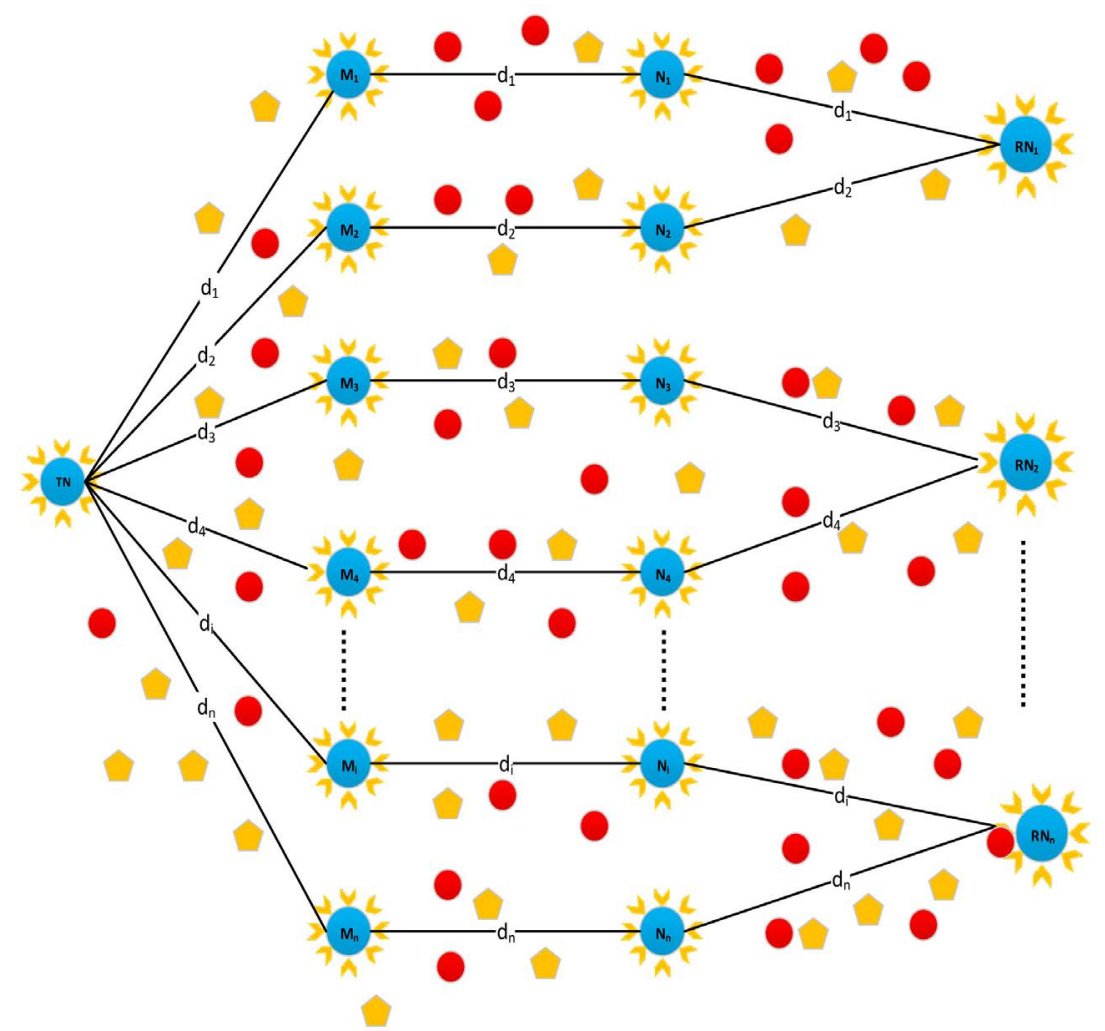

Figure 5. The system of MCD2 in the multipath. 
$\mathrm{RN}_{1}$. These information molecules can also arrive at the destination nanomachine $\mathrm{RN}_{2}$ by passing along the path $s_{3}$ or $s_{4}$. In addition, the TN releases some molecules can reach the $\mathrm{RN}_{\mathrm{n}}$ through $s_{i}$ or $s_{2 n}$ before one time slot. Therefore, the reliability of the multipath can be defined as $P_{m u l}$ which means the probability that at least one molecule from $\mathrm{TN}$ can be successfully received by $\mathrm{RN}_{1}, \mathrm{RN}_{2}, \mathrm{RN}_{3}, \cdots, \mathrm{RN}_{\mathrm{i}}$ or $\mathrm{RN}_{\mathrm{n}}$ before time $T$.

Firstly, we consider that the reliability of the case at least one information molecule from $\mathrm{TN}$ can be successfully received by $\mathrm{RN}_{1}$ passing along the path $s_{1}$ or $s_{2}$ can be defined as $P_{S_{1,2}}$. In this case, we can get the reliability $P_{S_{1,2}}$ for the two paths from the $\mathrm{TN}$ to the $\mathrm{RN}_{1}$ as follows:

$$
\begin{gathered}
P_{S_{1,2}}=1-\prod_{s_{k} \subseteq S}\left(1-P_{s_{k}}\right), k \in[1,2], k \in Z \\
P_{s_{k}}=\prod_{s_{k} \subseteq S} P_{i j}
\end{gathered}
$$

where $P_{i j}$ can be calculated by the Equation (10).

Then we can consider the reliability of the case that at least one information molecule from TN can be successfully received by $\mathrm{RN}_{2}$ passing along the path $s_{3}$ or $s_{4}$ can be defined as $P_{S_{3,4}}$. In this case, we can get the reliability $P_{S_{3,4}}$ for the two paths from the TN to the $\mathrm{RN}_{2}$ as follows:

$$
P_{S_{3,4}}=1-\prod_{s_{k} \subseteq S}\left(1-P_{s_{k}}\right), k \in[3,4], k \in Z
$$

where $P_{s_{k}}$ can be calculated by the Equation (20).

Finally, we compute the reliability of the case that at least one information molecule from $\mathrm{TN}$ can be successfully received by $\mathrm{RN}_{\mathrm{n}}$ passing along the path $S_{i}$ or $s_{2 n}$ can be represented by $P_{S_{i, 2 n}}$. Base on this case, we can get the reliability $P_{S_{i, 2 n}}$ for the two paths from the TN to the $\mathrm{RN}_{\mathrm{n}}$ as follows:

$$
P_{S_{i, 2 n}}=1-\prod_{s_{k} \subseteq S}\left(1-P_{s_{k}}\right), k \in[i, 2 n], k \in Z
$$

where $P_{s_{k}}$ can be calculated by the Equation (20).

Therefore, we can calculate the reliability of the case that at least one information molecule from $T N$ can be successfully received by $R_{1}, R N_{2}, R_{3}, \cdots, R_{i}$ or $\mathrm{RN}_{\mathrm{n}}$. According to our previous definition about the reliability of the multipath, $P_{m u l}$ can be calculated as follows Equation (23):

$$
P_{m u l}=P_{S_{1,2}}+P_{S_{3,4}}+P_{S_{4,5}}+\cdots+P_{S_{i, 2 n}} .
$$

\section{Simulation Experiment and Result Analysis}

In this section, we will via numerical analysis to obtain simulation results. This experiment runs on a Windows 10 (64-bit) operating system, a PC with a memory size of $8 \mathrm{~GB}$, and a CPU of i7-9700. Then, we use MATLAB software to do some simulation experiments. Firstly, we investigate the influence of different model parameters for the reliability of MCD2 in the single link. These parameters are given in Table 1 . Then we compare the reliability performance of different models between MCD2 and the existing molecular communication based 
Table 1. Simulation parameters.

\begin{tabular}{ccc}
\hline Symbol & Description & Unit \\
\hline$V$ & the drift velocity of medium & $\mathrm{um} / \mathrm{s}$ \\
$d$ & distance between two nanomachines & $\mathrm{um}$ \\
$D$ & the diffusion coefficient of environment & $\mathrm{um}^{2} / \mathrm{s}$ \\
$m$ & number of time slot & - \\
$M$ & number of molecules each time slot & - \\
\hline
\end{tabular}

on free diffusion. Furthermore, we also compare the reliability of MCD2 between multipath and single path.

\subsection{Single Link}

We consider that drift velocity of medium, the distance from TN to RN, diffusion coefficient, time slot numbers have effect on the reliability of MCD2 in the single link. Therefore, it is necessary to investigate how these parameters affect the reliability of MCD2.

\subsubsection{The Effect of Drift Velocity on the Reliability}

Here, we suppose that the diffusion coefficient $D=0.8 \mathrm{um}^{2} / \mathrm{s}$, time slot number $m=10$, diffusion distance $d=8 \mathrm{um}$, and then we can use the reliability model of MCD2 to analyze the influence of different drift velocity on the reliability of MCD2, Such as $v=0.8 \mathrm{um} / \mathrm{s}, v=0.85 \mathrm{um} / \mathrm{s}$ and $v=0.9 \mathrm{um} / \mathrm{s}$, Therefore, we can see that the reliability of MCD2 varies with different drift velocity from Figure 6.

As can be seen from Figure 6, the reliability of MCD2 varies with drift velocity. When the drift velocity of the medium is gradually increased, the reliability of MCD2 is significantly improved. This mainly depends on the drift velocity of the medium, which makes the information molecules fast transmission in the channel. These information molecules are less likely to accumulate in the channel and cause symbol interference, which reduces the ISI noise in the channel to some extent. Therefore, it can be inferred that when some information molecules are sensitive to delay, the drift velocity can be faster, the system delay is reduced, and the reliability of MCD2 also has been improved.

\subsubsection{The Effect of Distance on the Reliability}

It is supposed that the diffusion coefficient $D=0.8 \mathrm{um}^{2} / \mathrm{s}$, drift velocity $v=0.8$ $\mathrm{um} / \mathrm{s}$, and time slot number $m=10$. Then, we can use the reliability model of MCD2 to analyze the influence of different diffusion distance on the reliability. Such as $d=7.5 \mathrm{um}, d=8 \mathrm{um}$ and $d=8.5 \mathrm{um}$. Therefore, we can see that the reliability of MCD2 varies with diffusion distance from Figure 7.

It can be seen from Figure 7 that as the diffusion distance between TN and $\mathrm{RN}$ increases, the reliability of molecular communication gradually decreases. This is mainly because the longer the diffusion distance, the longer the information molecules released by the TN arrive at the RN. When this information 


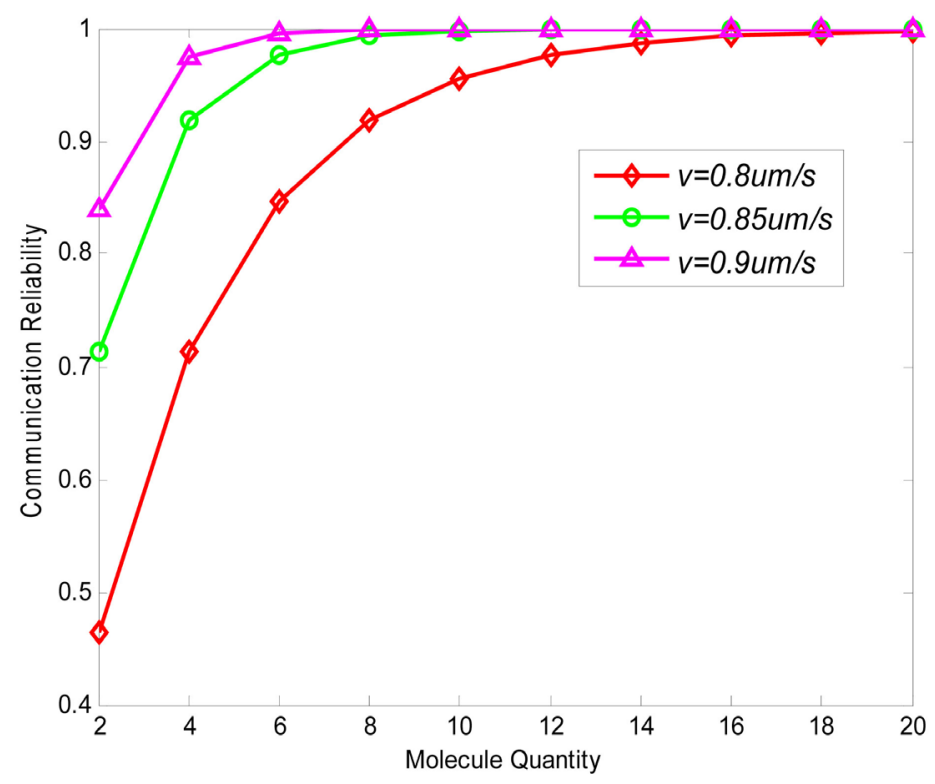

Figure 6. The reliability of molecular communication in different drift velocity.

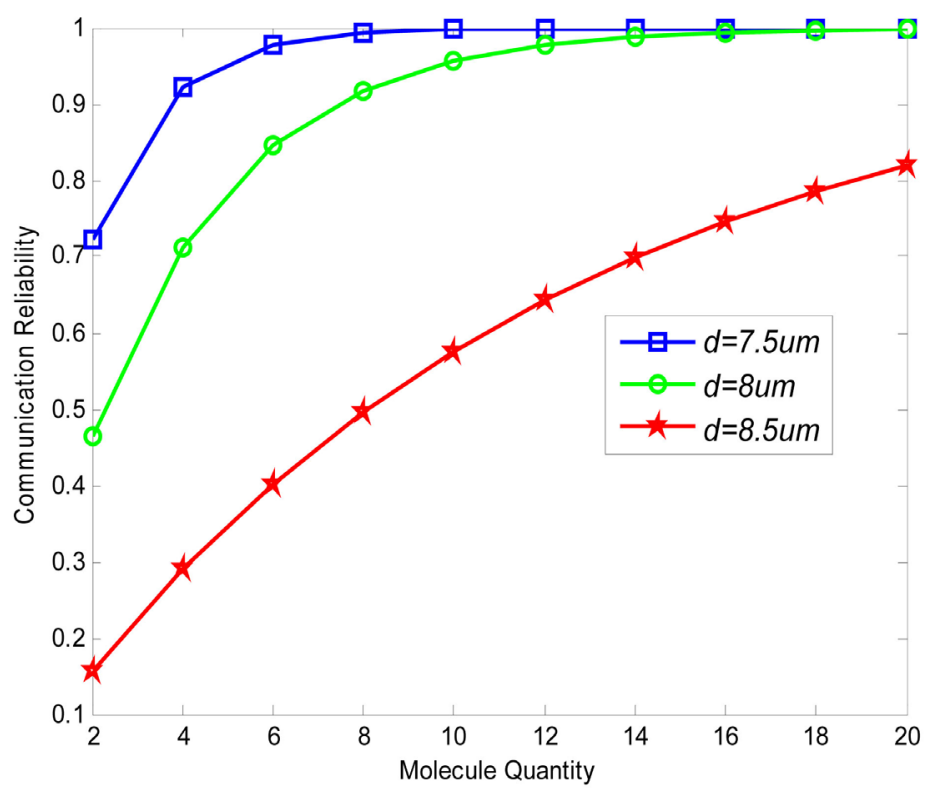

Figure 7. The reliability of molecular communication in different distance.

molecules diffuse in the channel, information molecules are prone to decay in the long time irregular motion. It makes the concentration of information molecules on the RN surface to gradually decrease, which leads to the problem of information decode error during the $\mathrm{RN}$ decodes information. Thus, the reliability of MCD2 is reduced to some extent.

\subsubsection{The Effect of Diffusion Coefficient for the Reliability}

It is assumed that drift velocity $v=0.8 \mathrm{um} / \mathrm{s}, d=8 \mathrm{um}$, and time slot number $m$ $=10$. Then we use the reliability model of molecular communication to study the influence of different diffusion coefficient on the reliability of MCD2. Such 
as $D=0.59 \mathrm{um}^{2} / \mathrm{s}, D=0.6 \mathrm{um}^{2} / \mathrm{s}, D=0.64 \mathrm{um}^{2} / \mathrm{s}$. Therefore, we can see that the reliability of MCD2 varies with different diffusion coefficient from Figure 8.

From Figure 8, it can be found that the reliability of the single link gradually increases as the number of molecules released in each time slot gradually increases. When the number of molecules increases to a certain upper threshold, the reliability of the link approaches 1 . In addition, when the diffusion coefficient of the environment is gradually increased, the reliability of molecular communication under a single link is maximized in a shorter time. The reason is that the larger the diffusion coefficient, the faster the information molecules released by the TN move in the channel, and the greater the probability of reaching the $\mathrm{RN}$ in the same time.

\subsubsection{The Effect of Time Slot for the Reliability}

We consider that drift velocity $v=0.8 \mathrm{um} / \mathrm{s}$, diffusion coefficient $D=0.8 \mathrm{um}^{2} / \mathrm{s}$, $d=0.8 \mathrm{um}$. Then, we can analyze the impact of different time slot length on the reliability of single link. For example, we take time slot $\tau=1 \mathrm{~s}, \tau=2 \mathrm{~s}$, and $\tau=3 \mathrm{~s}$, respectively.

From Figure 9, it can be seen that the reliability of molecular communication differs at different time slot length. Moreover, it can be concluded that as the length of time slot is larger, the reliability of molecular communication is higher. Therefore, we consider to extend the time slot properly within the acceptable delay range of both sides of the communication link. In this way, the influence of residual molecules in the channel on the decode of another symbol bit in the next time slot can be reduced, and the reliability of MCD2 can be further improved.

\subsubsection{The Effect of Retransmission Times on Reliability}

We presume that the diffusion coefficient of the environment $D=0.8 \mathrm{um}^{2} / \mathrm{s}, d=$ $8 \mathrm{um}$, drift velocity $V=0.8 \mathrm{um} / \mathrm{s}, \tau=1$, the number of time slot $m=10$. In the

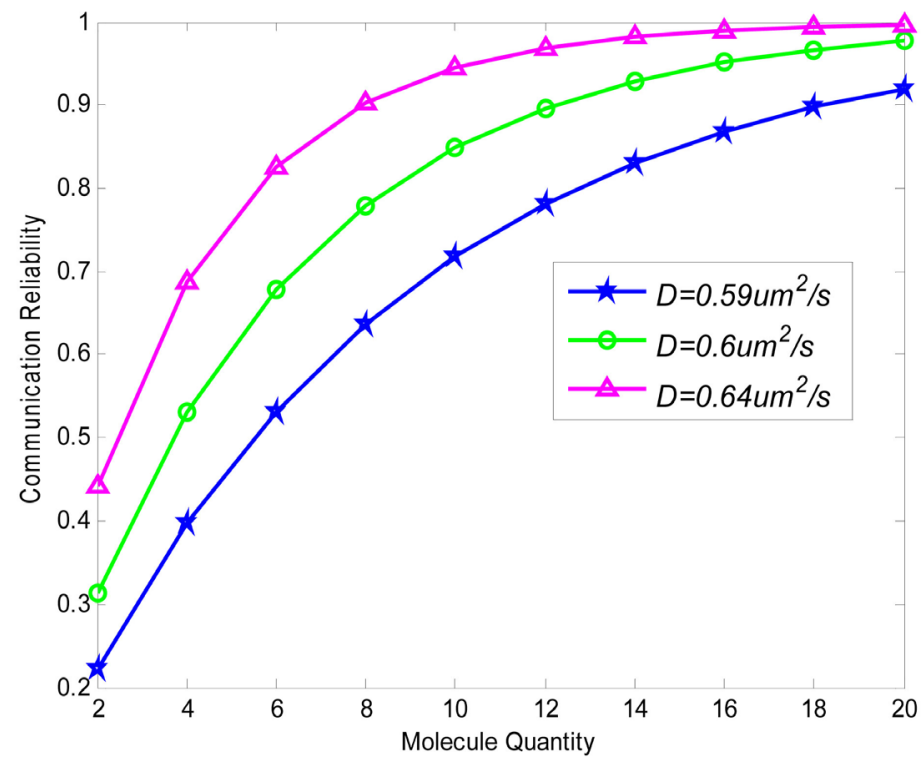

Figure 8. The reliability of molecular communication in different diffusion coefficient. 


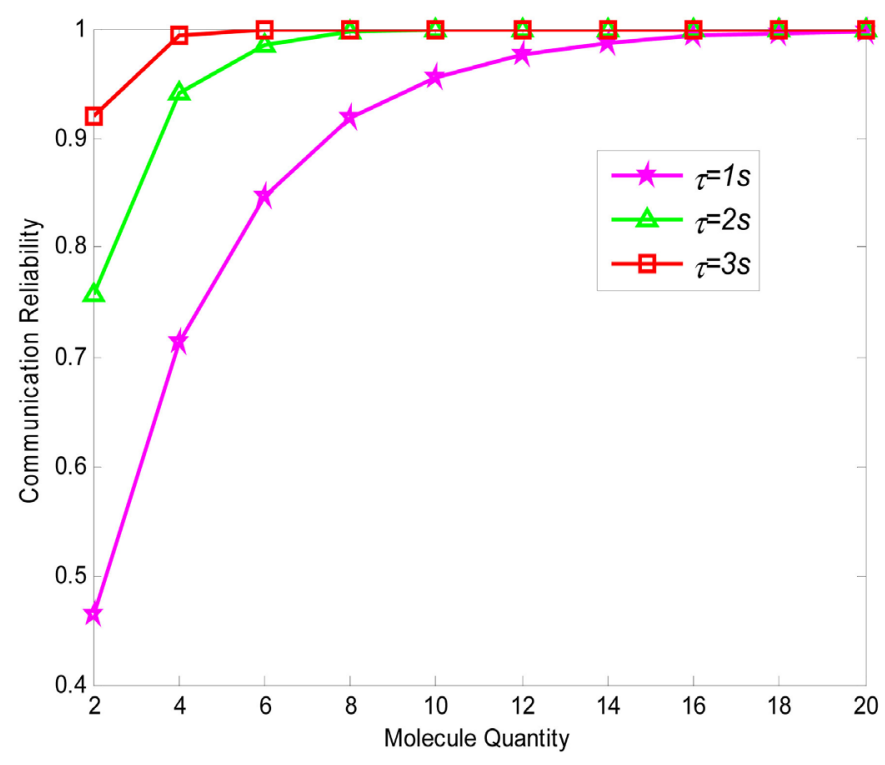

Figure 9. The reliability of molecular communication in different time slot.

process of transmission failure, a retransmission mechanism is used to ensure reliable transmission of information. Such as $C_{i j}=0, C_{i j}=1, C_{i j}=2, C_{i j}=3$. Therefore, we can see that the reliability of MCD2 varies with different retransmission times from Figure 10.

From Figure 10, we can see that the reliability of molecular communication is different under different retransmission times. Moreover, with the increase of retransmission times, the reliability of single link communication is higher. The reason is that in the case of transmission failure, the use of automatic retransmission mechanism can make the TN retransmit an equal number of molecules in a predetermined time, so that the information molecules transmitted by the $\mathrm{TN}$ are absorbed and decoded by the RN after multiple retransmissions, which improves the reliability of link communication to some extent. But at the same time, the communication link has a large delay. Therefore, in some links where the communication delay is not high, and the delay is within the acceptable range of both sides of the communication link, we can sacrifice time for high reliability.

\subsection{Performance Comparison of Different Reliability Model}

In the single link, the reliability model of MCD2 is compared with the existing model of molecular communication reliability based on free diffusion [8]. Under the condition that the parameters are set to the same value, we take the diffusion coefficient $D=0.59 \mathrm{um}^{2} / \mathrm{s}, d=5 \mathrm{um}, \tau=1$, drift velocity $v=0.6 \mathrm{um} / \mathrm{s}$, the number of time slot $m=10$ to compare the performance of reliability in different molecular communication reliability model. Therefore, we can analyze the performance of the reliability between reliability model of MCD2 and the existing model of molecular communication reliability based on free diffusion from Figure 11 . 


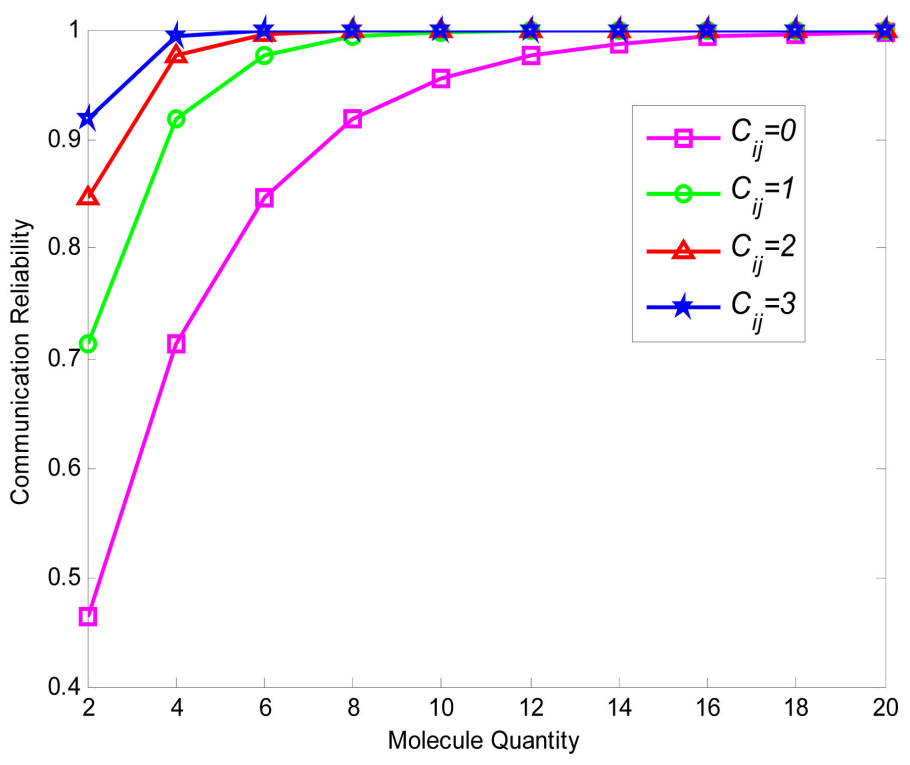

Figure 10. The reliability of molecular communication in different retransmission times.

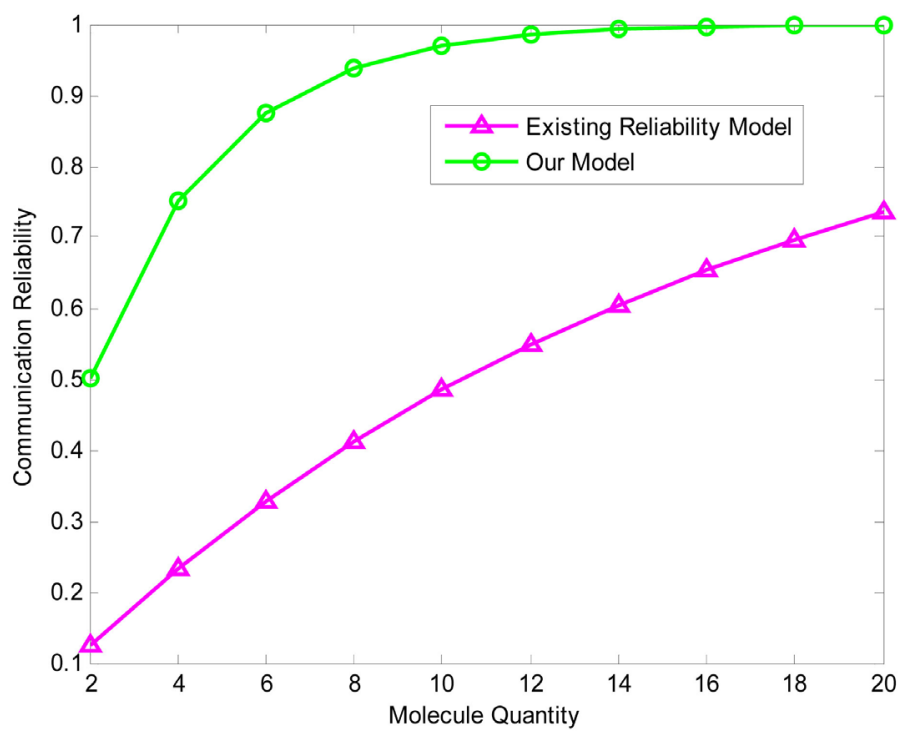

Figure 11. Performance comparison of different MC reliability models.

From Figure 11, it can be seen that the reliability of molecular communication increases with the increase of the number of molecules. When the number of molecules increases to a certain extent, the reliability of link will no longer increase and tend to 1 . Moreover, we also find that the reliability of MCD2 obviously higher than existing molecular communication based on free diffusion, which is mainly due to drift velocity accelerates information molecules reaching the $\mathrm{RN}$, thereby increasing the possibility that the $\mathrm{RN}$ receives the information molecules. Based on the above analysis, if we continue to use the existing free diffusion-based molecular communication reliability model to analyze the reliability of MCD2, the analysis of the reliability of the communication link will produce a higher error. 


\subsection{The Comparison of Reliability between Multipath and Single Path}

In this part, we mainly focus on comparing the reliability of MCD2 between multipath and single path. Firstly, we assume that diffusion coefficient $D=1$ $\mathrm{um}^{2} / \mathrm{s}, V=0.5 \mathrm{um} / \mathrm{s}$ and the number of time slot $m=10$ in the multipath and single path. Secondly, we take $n=3$ to indicate that there are three receiver nanomachines in the multipath. What's more, we also assume that the distance between the corresponding two nanomachines is equal and independent. The distance of each hop between two adjacent nanomachines is $d_{1}=4 \mathrm{um}$, $d_{2}=5 \mathrm{um}, d_{3}=6 \mathrm{um}, d_{4}=7 \mathrm{um}, d_{5}=8 \mathrm{um}, d_{6}=9 \mathrm{um}$ in six paths. As for the single path, we also suppose that $d_{1}=4 \mathrm{um}, d_{2}=5 \mathrm{um}$. Then we can use Equation (18) and Equation (23) to analyze the reliability of MCD2 in the single path and multipath.

Figure 12 shows that the reliability performance of MCD2 between multipath and single path. We can find that the reliability of the multipath obviously higher than the single path. This is mainly because the information molecules can easily reach the $\mathrm{RN}$ through multiple paths in the multipath, and the more information molecules can be received by RN. Therefore, the reliability of information transmission in the multipath is higher than that of single path.

\section{Conclusion}

In this paper, we propose a reliability model of MCD2 to investigate the reliability of different topologies between $\mathrm{TN}$ and $\mathrm{RN}$. In the case of transmission failure, a retransmission mechanism is used to ensure the reliability of information transmission. Furthermore, it can be concluded by numerical analysis that with the increase of drift velocity, diffusion coefficient, retransmission times, the length of time slot, and the decrease of diffusion distance, the reliability of MCD2

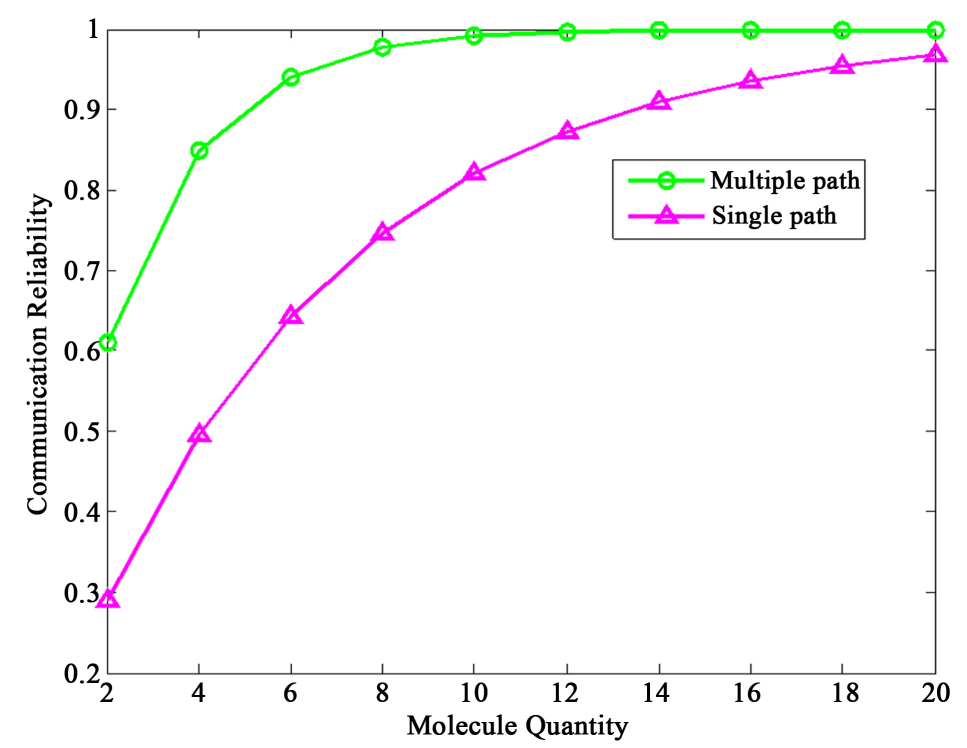

Figure 12. The comparison of reliability between multipath and single path. 
can be improved. At the same time, we also find that our proposed model of reliability is superior to the existing reliability model of molecular communication based on free diffusion in analyzing the reliability of MCD2.

\section{Conflicts of Interest}

The authors declare no conflicts of interest regarding the publication of this paper.

\section{References}

[1] Grillo, G., Akyildiz, I.F., Jornet, J.M. and Pierobon, M. (2011) Nanonetworks: A New Frontier in Communications. Communications of the ACM, 54, 84-89. https://doi.org/10.1145/2018396.2018417

[2] Nakano, T. and Liu, J.Q. (2010) Design and Analysis of Molecular Relay Channels: An Information Theoretic Approach. IEEE Transactions on NanoBioscience, 9, 213-221. https://doi.org/10.1109/TNB.2010.2050070

[3] Llatser, I., Cabellos-Aparicio, A. and Alarcon, E. (2012) Networking Challenges and Principles in Diffusion-Based Molecular Communication. IEEE Wireless Communications, 19, 36-41. https://doi.org/10.1109/MWC.2012.6339470

[4] Balasubramaniam, S. and Lio, P. (2013) Multi-Hop Conjugation Based Bacteria Nanonetworks. IEEE Transactions on NanoBioscience, 12, 47-59. https://doi.org/10.1109/TNB.2013.2239657

[5] Akyildiz, I.F., Brunetti, F. and Blázquez, C. (2008) Nanonetworks: A New Communication Paradigm. Computer Networks, 52, 2260-2279. https://doi.org/10.1016/j.comnet.2008.04.001

[6] Malak, D. and Akan, O. (2014) Communication Theoretical Understanding of Intra-Body Nervous Nanonetworks. IEEE Communications Magazine, 52, 129-135. https://doi.org/10.1109/MCOM.2014.6807957

[7] Frank, W. and Balasubramaniam, S. (2013) Reliability and Delay Analysis of Multihop Virus-Based Nanonetworks. IEEE Transactions on Nanotechnology, 5, 674-684. https://doi.org/10.1109/TNANO.2013.2268389

[8] Cheng, Z., Zhu, Y.H., Chi, K.K. and Li, Y.J. (2016) Reliability and Delay Analysis of Multicast in Binary Molecular Communication. Nano Communication Networks, 9, 17-27. https://doi.org/10.1016/j.nancom.2016.07.002

[9] Einolghozati, A., Sardari, M. and Fekri, F. (2016) Networks of Bacteria Colonies: A New Framework for Reliable Molecular Communication Networking. Nano Communication Networks, 7, 17-26. https://doi.org/10.1016/j.nancom.2015.01.003

[10] Tepekule, B., Pusane, A.E., Kuran, M.S. and Tugcu, T. (2015) A Nove Pre-Equalization Method for Molecular Communication via Diffusion in Nanonetworks. IEEE Communications Letters, 19, 1311-1314. https://doi.org/10.1109/LCOMM.2015.2441726

[11] Shih, P., Lee, C., Yeh, P. and Chen, K. (2013) Channel Codes for Reliability Enhancement in Molecular Communication. IEEE Journal on Selected Areas in Communications, 31, 856-867. https://doi.org/10.1109/JSAC.2013.SUP2.12130018

[12] Leeson, M.S. and Higgins, M.D. (2012) Forward Error Correction for Molecular Communications. Nano Communication Networks, 3, 161-167. https://doi.org/10.1016/j.nancom.2012.09.001

[13] Lu, P.F., Wu, Z.Q. and Liu, B. (2017) A Vertical Channel Model of Molecular 
Communication and Its Test-Bed. EAI Endorsed Transactions on Pervasive Health and Technology, 9, 1-8. https://doi.org/10.4108/eai.21-3-2017.152390

[14] Lu, P.F., You, Y., Liu, B. and Wu, Z.Q. (2015) A Vertical Channel Model of Molecular Communication based on Alcohol Molecules. 9th EAI International Conference on Bio-Inspired Information and Communications Technologies, New York, 3-5 December 2015, 157-162. https://doi.org/10.4108/eai.3-12-2015.2262444

[15] Crank, J. (1979) The Mathematics of Diffusion. Oxford University Press, Oxford.

[16] Kadloor, S., Adve, R.S. and Eckford, A.W. (2012) Molecular Communication Using Brownian Motion with Drift. IEEE Transactions on NanoBioscience, 11, 89-99. https://doi.org/10.1109/TNB.2012.2190546 